\title{
On Convergence Fields of Some Regular Matrix Transformations
}

\author{
Zakawat U. Siddiqui, Dauda I. Nkuno
}

\begin{abstract}
Some properties of convergence field of regular matrix transformation have already been established.The study of $\sigma$-porous sets has also been carried out. In this paper, we establish the conditions under which the convergence field $F(A)$ of a regular transformation $A$ is porous in $S(A)$, the set of A-limitable real sequence.We also determine the conditions under which $F(A)$ dense in $S(A)$. Further, we prove that the convergence field ofvarious matrix transformation are close inS(A).

Mathematics Classification: 40D09, 46B45
\end{abstract}

Index Terms-Convergence field, Dense sets,Porosity, Regular and Irregular Matrix Transformations.

\section{INTRODUCTION}

The concept of porosity in $\mathbb{R}$ was used by Denjoy [1] in 1920 under a different nomenclature. He proved that if $P$ is a perfect nowhere dense subset of $\mathbb{R}$, then the set of points in $P$ at which $P$ is not strongly bilaterally porous is a first category subset of $P$. Dolženko [2] in 1967 introduced the notion of $\sigma$-porous sets and applied the notion to cluster sets of functions while in the year 1976, Šalát [3] showed the usefulness of the theorem of the discontinuity points of functions of the first Baire category in the study of the convergence field of a regular matrix method. Renfro [4] proved two strong versions of Denjoy statement for an arbitrary metric space. Kostyrko [5]in 2004 discussed some properties of the convergence field of regular matrix transformation of bounded sequences of real numbers. Visnyai [6] in 2006 proved a generalization of Steinhaus theorem for sequences of a Banach space and showed that the result of Šalàt [3] and Kostyrko [5] can be generalized for a space of sequences of element of a $\operatorname{Banach} \operatorname{space}(X,\|\|$.$) .$ Later Kostyrko [7] in 2008 showed that the convergence field $F(A)$ of a regular matrix transformation is a $\sigma$-porous set in the metric space $S(A)$ endowed with Fréchet topology. Peter Letavaj [8] in 2012 showed that the convergence field $F(A)$ of a regular matrix transformation is a very porous set in $\mathrm{S}$.

\section{METHOD AND MATERIAL}

In this paper we shall make use of the following material and known results.

Definition 2.1[9]: Suppose $(X, \rho)$ is a metric space, $T \subset X$. Let $x \in X, \delta>0$ and

$$
B(x, \delta)=\{y \in X: \rho(x, y)<\delta\}
$$

Zakawat U. Siddiqui, Department of Mathematical Sciences, University of Maiduguri, Maiduguri, Nigeria

Dauda I. Nkuno, Department of Mathematical Sciences, University of Maiduguri, Maiduguri, Nigeria.
Then, we put

$\lambda(x, \delta, T)=\sup \{t>0: \exists z \in B(x, \delta)$ such that $B(z, t)$

$$
\subset B(x, \delta) \text { and } B(z, t) \cap T=\emptyset)\}
$$

If such $t>0$ does not exist, we put $\lambda(x, \delta, T)=0$.

The numbers

and

$$
\underline{p}(x, T)=\lim _{\delta \rightarrow 0} \inf \frac{\lambda(x, \delta, \mathrm{T})}{\delta}
$$

$$
\bar{p}(x, \mathrm{~T})=\lim _{\delta \rightarrow 0} \sup \frac{\lambda(x, \delta, \mathrm{T})}{\delta}
$$

are called lower and upper porosity of the set $T$ at $x$. If $\bar{p}(x, \mathrm{~T})>0$, then $T$ is porous at $x$ and if, for each $x \in \mathrm{X}$, $\bar{p}(x, \mathrm{~T})>0$ holds, then $\mathrm{T}$ is porous in $X$. If $p(x, T)>0$, then $T$ is very porous at $x$ and if for each $x \in \overline{\mathrm{X}}, \underline{p}(x, T)>$ 0 holds, then $T$ is very porous in $X$.

Note that when a (divergent) sequence, say $\left\{x_{n}\right\}$ is acted upon by an infinite matrix $A=\left(a_{n k}\right)$ say, the resultant sequence $\left\{t_{n}\right\}$, given by

$$
t_{n}=\sum_{k=1}^{\infty} a_{n k} x_{k}
$$

may exist. The set of all such sequences obtained for which the series in (1.1) converge is called the convergence field of the given matrix.

Therefore, if suppose $S$ denotes the set of all the sequences of real or complex numbers and $S(A)$ is the set of all those sequences for which the sequence given by the series in (1.1) exists, then the convergence field of the matrix $A$ is given by

$$
\mathrm{F}(\mathrm{A})=\left\{x \in S(A) \mid t \exists \lim _{n \rightarrow \infty} t_{n}=\lim _{n \rightarrow \infty} \sum_{k=1}^{\infty} a_{n k} x_{k}\right\}
$$

\section{(1.2)}

It is well-known that the necessary and sufficient conditions under which the matrix method $(A)$ is regular are given as:

$$
\begin{array}{ll}
\text { i. } & \sum_{k=1}^{\infty}\left|a_{n k}\right|<\infty \text { for each } n=1,2, \ldots \\
\text { ii. } & \lim _{n \rightarrow \infty} a_{n k}=0 \\
\text { iii. } & \lim _{n \rightarrow \infty} \sum_{k=1}^{\infty} a_{n k}=1
\end{array}
$$

Theorem 2.1 [5]: Let $(S, \rho)$ be the space of all bounded sequence of real or complex numbers endowed with the metric ' $\rho$ '. Then, the convergence field F(A), is a very porous set in $S(A)$.

Theorem 2.2 [7]: Let $\mathrm{A}$ be a regular matrix, then the convergence field $F(A)$ of the matrix $(A)$ is a $\sigma$-porous set in $S(A)$.

Theorem 2.3 [3]:Let $T=\left(a_{n k}\right)$ and let $(T)$ be a regular method. Let $\mathrm{Y}$ be a metric space and $Y_{1}(T)$ be the set of all T-limitable sequences of $\mathrm{Y}$. Let $M_{k}, \mathrm{k}=1,2, \cdots$, be a non-void set of complex numbers. Let us suppose that

(j) $\sup _{k=1,2 \ldots} \operatorname{diam} M_{k}<+\infty$, 


\section{On Convergence Field of Some Regular Matrix Transformation}

(ii) There exist two sequences

$y^{\prime}=\left(\eta_{k}^{\prime}\right)_{k=1}^{\infty} \in Y_{1}(T) \quad$ and $\quad y^{\prime \prime}=\left(\eta_{k}^{\prime \prime}\right)_{k=}^{\infty} \in Y_{1}(T)$ such that $\left\{\eta_{k}^{\prime \prime}-\eta_{k}^{\prime \prime}\right\}$ is a convergence sequences andthat $\lambda=$ $\left(\eta_{k}^{\prime \prime}-\eta_{k}^{\prime}\right) \neq 0$

Then the set $Y_{1}(T)$ is a dense set (in $Y$ ) of the first Baire category.

\section{MAIN RESULTS}

Definition 3.1: Let $A=\left(a_{n k}\right)$ be any infinite matrix and $x=\left(x_{k}\right)$ be any infinite sequence. Then, the convergence field $F(A)$ is close in $S(A)$ if $\forall x \in S(A) \backslash F(A)$ and $\forall \delta>0, B(x, \delta) \cap F(A)=\emptyset$.

Theorem 3.1: Let $A=\left(a_{n k}\right)$ be any infinite matrix and $\mathrm{S}$ be the space of all sequences of real or complex numbers. Then, $F(A)$ is porous in $S(A)$ if and only $A=\left(a_{n k}\right)$ is irregular.

Proof. Suppose $F(A)$ is porous in $S(A)$. Then by definition 2.1, we have

$$
\begin{aligned}
& P(x, F(A))>0 \\
& \Rightarrow \lim _{\delta \rightarrow 0^{+}} \sup \frac{\lambda(x, \delta, F(A))}{\delta}>0 \\
& \Rightarrow \lambda(x, \delta, F(A))>0
\end{aligned}
$$

This implies that the set

$\{t>0: \exists y \in B(x, \delta): B(y, t) \subset$

$B x, \delta$ and $B y, t \cap F A=\varnothing \neq \varnothing$.

Therefore, the number $\sup \{t>0 ; \exists y \in B(x, \delta) ; B(y, t)$

$$
\subset B(x, \delta) \text { and } B(y, t) \cap F(A)=\emptyset\}>0
$$

$\Rightarrow$ there exists $t>0$ such that $B(y, t) \cap F(A)=\emptyset$

$\Rightarrow B(y, t) \subset S(A) \backslash F(A)$

$\Rightarrow$ that not all the series in 1.1 which exists converges.

$\Rightarrow A=\left(a_{n k}\right)$ is irregular

Conversely, suppose that $A=\left(a_{n k}\right)$ is irregular. Then clearly, not all the series in 1.1 converge and therefore, $S(A) \backslash F(A) \neq \varnothing$. Thus for all $x \in S(A) \backslash F(A)$, there exist a number $\delta>0$ such that $B(x, \delta) \subset S(A)$. Further, there exists $y \in B(x, \delta)$ such that for some numbers

$t_{i}>0, B\left(y, t_{i}\right) \subset B(x, \delta)$ and $B\left(y, t_{i}\right) \cap F(A)=\emptyset, i=$ $1,2,3, \ldots$.

Therefore, there is a set

$\{t>0 ; \exists y \in B(x, \delta) ; B(y, t) \subset B(x, \delta)$ and $B(y, t) \cap F(A)$

$$
=\emptyset\} \neq \emptyset
$$

Hence the number

$$
\begin{aligned}
\sup \{t>0 ; \exists y \in & B(x, \delta) ; B(y, t) \\
& \subset B(x, \delta) \text { and } B(y, t) \cap F(A)=\emptyset\}>0 \\
\lambda(x, \delta, F(A))= & \sup \{t>0 ; \exists y \in B(x, \delta) ; B(y, t) \\
& \subset B(x, \delta) \text { and } B(y, t) \cap F(A)=\emptyset\}>0 \\
\Rightarrow & \lim _{\delta \rightarrow 0^{+}} \sup \frac{\lambda(x, \delta, F(A))}{\delta}>0 \\
\Rightarrow & P(x, F(A))=\lim _{\delta \rightarrow 0^{+}} \sup \frac{\lambda(x, \delta, F(A))}{\delta}>
\end{aligned}
$$

0

$$
\Rightarrow P(x, F(A))>0
$$

Hence $F(A)$ is porous in $S(A)$ at all points $x \in$ $S(A) \backslash F(A)$.

Theorem 3.2; If $A=\left(a_{n k}\right)$ is any infinite matrix and $\mathrm{S}$ is the space of all sequences of real or complex numbers. Then, $F(A)$ is dense in $S(A)$ if and only if $F(A)$ is not porous in $S(A)$.
Proof.We prove this theorem by contradiction. Suppose that $F(A)$ is porous in $S(A)$, then, by definition 2.1, we have;

$$
P(x, F(A))>0
$$

$\Rightarrow \lim _{\delta \rightarrow 0^{+}} \sup \frac{\gamma(x, \delta, F(A))}{\delta}>0$

$\Rightarrow \gamma(x, \delta, F(A))>0$

This implies that the set

$\{t>0: \exists y \in B(x, \delta): B(y, t) \subset B(x, \delta)$ and $B(y, t) \cap F(A)$

$$
=\emptyset\} \neq \varnothing
$$

And therefore the number

$\Rightarrow \sup \{t>0 ; \exists y \in B(x, \delta) ; B(y, t)$

$$
\subset B(x, \delta) \text { and } B(y, t) \cap F(A)=\emptyset\}>0
$$

$\Rightarrow$ there exists $t>0$ such that $B(y, t) \cap F(A)=\emptyset$

$\Rightarrow B(y, t) \subset S(A) \backslash F(A)$

$\Rightarrow A=\left(a_{n k}\right)$ is irregular contradicting the fact that $A=\left(a_{n k}\right)$ is regular.

Hence, $t>0$ such that $B(y, t) \subset S(A) \backslash F(A)$ does not exist

$\Rightarrow S(A) \backslash F(A)=\varnothing$

This implies that the set

$\{t>0: \exists y \in B(x, \delta): B(y, t) \subset B(x, \delta)$ and $B(y, t) \cap F(A)$

$$
=\varnothing\}=\varnothing
$$

This implies that the number

$\Rightarrow \sup \{t>0: \exists y \in B(x, \delta): B(y, t)$

$\Rightarrow \gamma(x, \delta, F(A))=0$

$$
\subset B(x, \delta) \text { and } B(y, t) \cap F(A)=\emptyset\}=0
$$

$\Rightarrow \lim _{\delta \rightarrow 0^{+}} \sup \frac{\gamma(x, \delta, F(A))}{\delta}=0$

$P(x, F(A))=0$

$\Rightarrow F(A)$ is not porous in $S(A)$

$\Rightarrow$ that for any point $y \in S(A)$ and $t>0, B(y, t) \cap F(A) \neq$ $\emptyset$.

Hence, $F(A)$ is everywhere dense in $S(A)$ and $\overline{F(A)}=$ $S(A)$.

Conversely, suppose $F(A)$ is dense in $S(A)$. Then, for any $y \in S(A)$ there exist always a number $t>0$ such that $B(y, t) \cap F(A) \neq \varnothing$

$\Rightarrow S(A) \backslash F(A)=\emptyset$

$\Rightarrow \nexists t>0 \forall x \in S(A)$ and $\delta>0$ such that for any $y$ $\in B(x, \delta)$

$B(y, t) \subset B(x, \delta)$ and $B(y, t) \cap F(A)=\emptyset$

Since $t>0$ does not exist, then,

$\Rightarrow \gamma(x, \delta, F(A))=0$

$\Rightarrow \lim _{\delta \rightarrow 0^{+}} \sup \frac{\gamma(x, \delta, F(A))}{\delta}=0$

$\Longrightarrow P(x, F(A))=0$

$\Rightarrow F(A)$ is not porous in $S(A)$ and hence the proof.

Theorem 3.3: Let $A=\left(a_{n k}\right)$ be any infinite matrix and $S$ be the space of all sequences of real or complex numbers. Then, $F(A)$ is close in $S(A)$ if and only if $F(A)$ is porous in $S(A)$.

Proof:

Suppose that $F(A)$ is porous in $S(A)$. Then, Theorem 3.1, implies that $A=\left(a_{n k}\right)$ is irregular and therefore,

$$
P(x, F(A))>0
$$

$\bar{P}(x, F(A))=\underline{P}(x, F(A))$

$\Rightarrow \bar{P}(x, F(A))=\lim _{\delta \rightarrow 0^{+}} \sup \frac{\lambda(x, \delta, F(A))}{\delta}>0$ 
$\Rightarrow \lambda(x, \delta, F(A))>0$

This implies that the set

$\{t>0: \exists y \in B(x, \delta): B(y, t) \subset B(x, \delta)$ and $B(y, t) \cap F(A)$

$$
=\emptyset\} \neq \emptyset
$$

$\Rightarrow \sup \{t>0 ; \exists z \in B(x, \delta) ; B(z, t)$

$\subset B(x, \delta)$ and $B(z, t) \cap F(A)\}>0$

$\Rightarrow$ there exist $t>0$ for some $z$

$\in B(x, \delta)$ such that $B(z, t) \cap F(A)=\emptyset$

$\Longrightarrow S(A) \backslash F(A) \neq \emptyset$

$\Longrightarrow \forall z \in S(A) \backslash F(A) \exists \varepsilon>0 \mathrm{z} \in \mathrm{SA} \backslash \mathrm{FA} \exists \varepsilon>0$ such that $B(z, \varepsilon) \subset S(A) \backslash F(A)$

$\Rightarrow S(A) \backslash F(A)$ is open. Hence $F(A)$ is closed in $S(A)$.

Conversely, supposed that $F(A)$ is closed in $S(A)$. Then, $S(A) \backslash F(A)$ is open in $S(A)$.

Hence, $\forall z \in S(A) \backslash F(A)$, there exist a number say $\varepsilon>0$ such that $B(z, \varepsilon) \subset S(A) \backslash F(A)$. Thus, $\forall x \in S(A)$, there exist a number $\delta>0$ such that $B(z, \varepsilon) \subset B(x, \delta), B(z, \varepsilon) \cap$ $F(A)=\emptyset$.

This implies that the set $\{\varepsilon>0 ; \exists z \in B(x, \delta) ; B(z, \varepsilon) \subset$ $B x, \delta$ and $B z, \varepsilon \cap F A=\varnothing \neq \varnothing$.

$\Longrightarrow$

$\sup \{\varepsilon>0 ; \exists z \in B(x, \delta) ; B(z, \varepsilon) \subset$

$B x, \delta$ and $B z, \varepsilon \cap F A>0$.

$\Rightarrow \lambda(x, \delta, F(A))>0$.

$$
\begin{aligned}
P(x, F(A))=\lim _{\delta \rightarrow 0^{+}} & \sup \frac{\lambda(x, \delta, F(A))}{\delta} \\
& =\lim _{\delta \rightarrow 0^{+}} \inf \frac{\lambda(x, \delta, F(A))}{\delta}> \\
& \Rightarrow P(x, F(A))>0
\end{aligned}
$$

$\Longrightarrow F(A)$ is porous in $S(A)$ at all the points $x \in S(A) \backslash$ $F(A)$ and hence the theorem.

\section{REFERENCES}

[1] A. Denjoy, "Lecçns sur le Calcul des Coefficients d'une Série Trigonométrique (Part II)", Gauthier-Villars, 1941

[2] E. P. Dolženko, "Boundary properties of arbitrary functions", Izv. Akad. Nauk SSSR Ser. Mat. 31, 1967, 3 - 14

[3] T. Šalàt,., "On convergence fields of regular matrix transformations ", Czechoslovak Mathematical journal, vol. .261976, No. 101,613-627

[4] D. L. Renfro, "Porosity, nowhere dense sets and a theorem of Denjoy", Real Analysis Exchange, 21(2), , 1995-96, 572 - 581.

[5] P. Kostyrko, "Convergence field of regular matrix transformations", Tatra Mount. Math Publ., 28, 2004, 153-157.

[6] T. Visnyai, "Convergence field of regular matrix transformations of sequences of elements of Banach spaces", Miskolc Mathematics notes, Vol. 7, 2006, No 1, pp. $101-108$

[7] P. Kostyrko, "Convergence fields of regular matrix transformations 2", Tatra Mt. Math. Publ. 40, 2008, 143-147.

[8] [8] P. Letavaj," "Convergence field of Abel's summation method", Math. Slovaca, 62, 2012, No. 3, 525-530

[9] L. Zajíček,, "On $\sigma$-porous sets in abstract spaces", Abstract and Appld Analysis, 2005, 509 - 534. 